\title{
Acrometastasis as the initial presentation of bronchogenic carcinoma
}

\author{
Kamonpun Ussavarungsi, MD, Brenda Watkins, MSSW, Michael Phy, DO
}

\begin{abstract}
Metastasis to the digits occurs infrequently and has a nonspecific clinical presentation. We report a patient who had persistent swelling and ulceration involving his right thumb nail. He did not have a prior diagnosis of cancer, and this delayed his eventual diagnosis of non-small cell cancer of the lung with acrometastasis. He was treated with amputation and other palliative measures. Physicians should include acrometastasis in the differential diagnosis in patients with persistent non-healing digital injuries or ulcers. These patients usually have limited treatment options and a very poor prognosis.
\end{abstract}

Key words: ncarcinoma, bronchogenic; neoplasm metastasis; bone neoplasm

\section{INTRODUCTION}

Acrometastasis occur infrequently, and its presentation mimics an infectious or inflammatory disease. Therefore, this diagnosis is often delayed, especially in cases in which a primary cancer is not suspected. Our patient presented with metastasis to the hand from an undiagnosed non-small cell lung cancer and demonstrates that acrometastasis must be included in the differential diagnoses when treating a non-healing digital lesion.

\section{Case Presenttion}

An 85-year-old Hispanic man presented with a swollen and small "sore-like" area on his right thumb which had been present for approximately four months. When this began to enlarge, he sought care from his physician and was treated multiple times for soft tissue infection. The antibiotic therapy did not improve the patient's symptoms, and he continued to have increased swelling, erythema, tenderness, and

Corresponding author: Kamonpun Ussavarungsi Contact Information: Ussavarungsi.Kamonpun@mayo.edu DOI: 10.12746/swrccc2013.0101.010

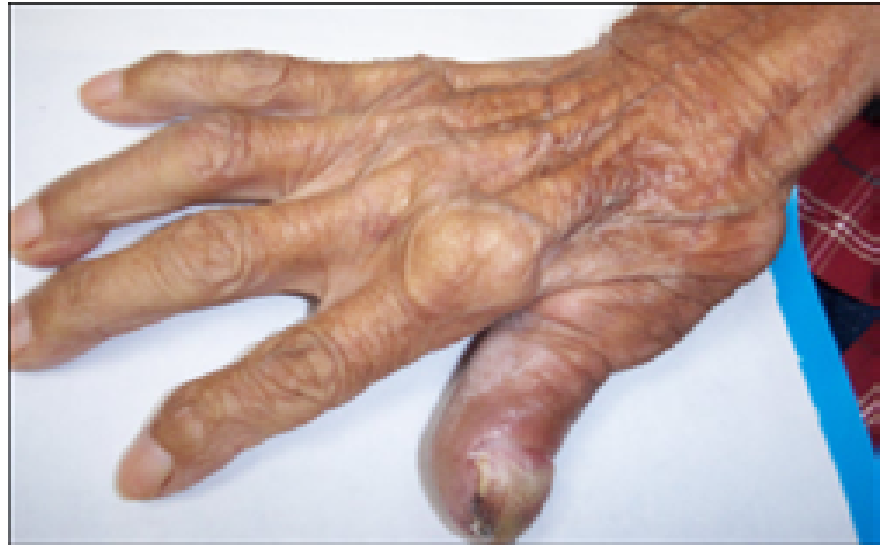

Figure 1. Photograph of right thumb with a swollen raised nail.

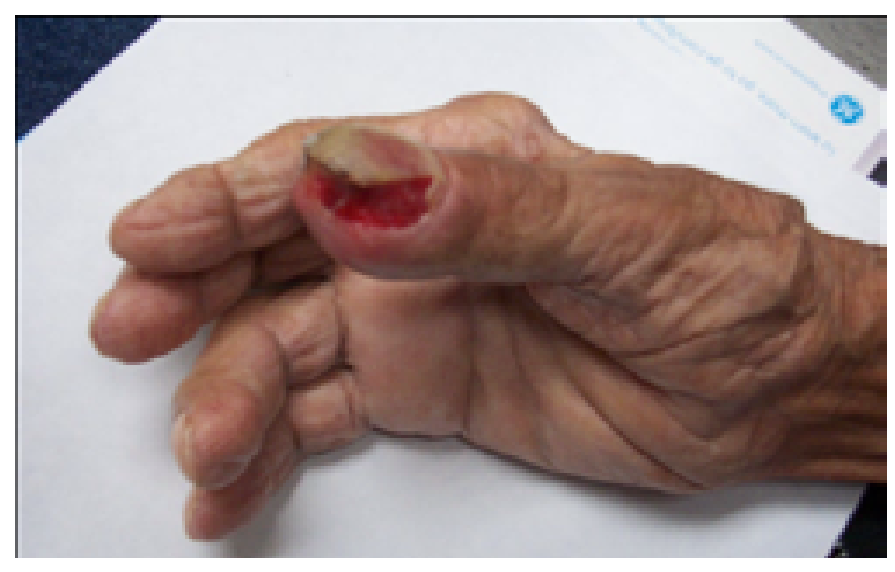

Figure 2. Photograph of right thumb showing ulceration below the nail. 
enlargement of the tip of the thumb. Eventually his nail lifted off the nail bed and the distal phalanx of his thumb bore a fungating mass. At the time, he de-

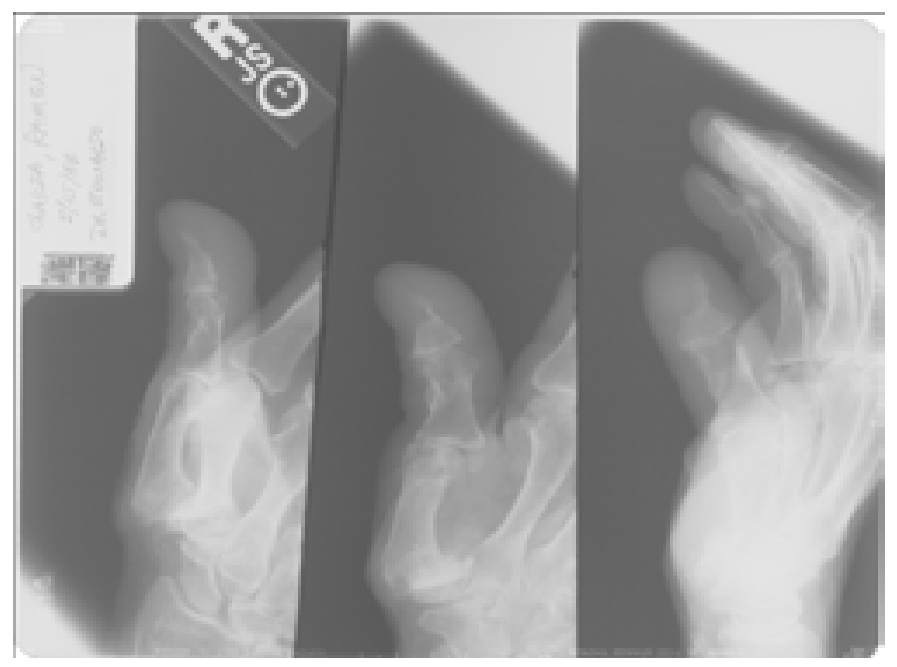

Figure 3. Plain X-ray showing loss of bone in the distal thumb.

nied fever and chills, had no other specific symptoms, and related no history of trauma. His physical examination revealed normal vital signs. His right thumb showed a large fungating mass growing from the nail bed and oozing clear serous material (Figures 1, 2). Plain films of the right thumb showed a destructive bone lesion involving the distal phalanx with its distal lateral aspect measuring 13.8 by $7.7 \mathrm{~mm}$, consistent with an osteolytic metastatic bone lesion (Figure 3 ). The pathologic report of a biopsy of the right thumb revealed infiltration of fibrous tissue by nests of cytologically malignant cells with focal gland formation on deeper sections compatible with poorly differentiated adenocarcinoma. The immunophenotype was positive for CKAE1/AE3 and CK7 and negative for S100 and CD20. It was strongly positive for TTF1 which is consistent with a primary lung cancer.

Prior to this evaluation, he reported no change in his health except for some shortness of breath with activity and decreased appetite with a 15 pound weight loss over several months. The patient smoked tobacco, approximately one cigarette per week. Additional physical examination revealed no cervical lymphadenopathy. Examination of the lungs revealed decreased breath sounds, dullness to percussion, and decreased tactile fremitus on the right side. This additional history and physical examination information led to imaging studies of the chest. A chest X-ray

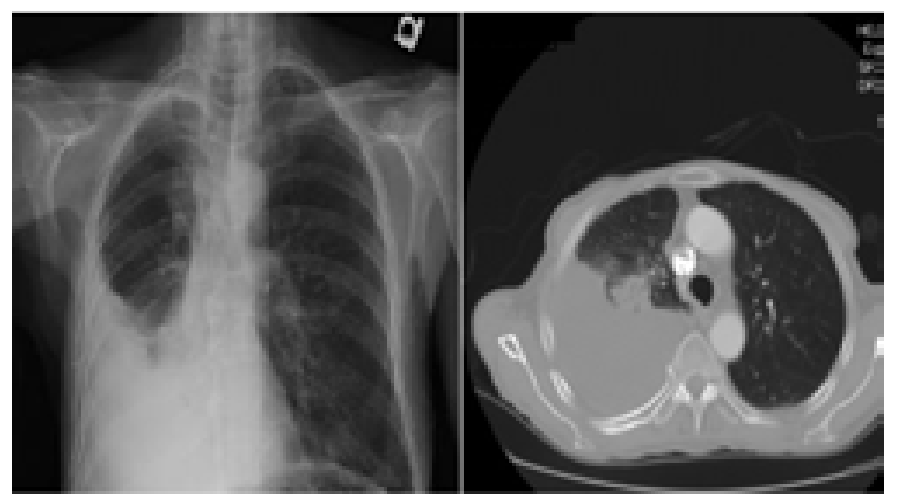

Figure 4. Plain chest X-ray showing a large right pleural effusion. The CT scan reveals a right pleural effusion and a tissue density above the effusion.

showed a right suprahilar patchy nodular mass measuring 3.8 by $3.3 \mathrm{~cm}$, mediastinal shift to the right, and a large right pleural effusion (Figure 4). CT scan of the chest showed small mediastinal nodes in the pretracheal, retrocaval, and subcarinal stations and a small left hilar node. A 2.5 by $2.3 \mathrm{~cm}$ nodular infiltrate was present in the right upper lobe. A large right pleural effusion was present. The patient was subsequently diagnosed with non-small cell lung cancer with right thumb metastasis.

\section{Discussion}

Based on the current literature, bone metastases from malignancy are frequent, but metastases to the extremities, especially the hands, are rare. Common sites of origin are lung, prostate, breast, and liver. $^{3} \quad$ Bone metastasis can occur in up to $70 \%$ of patients with advanced breast or prostate cancer and $15-30 \%$ of patients with carcinoma of the lung, coIon, stomach, bladder, uterus, rectum, thyroid or kidneys. ${ }^{3}$ Common sites of deposit are usually located in the axial or proximal appendicular bone, especially the spine. ${ }^{1}$ Pelvis, femurs, and ribs are the next most common sites. Approximately $0.1 \%$ of all metastatic osseous disease involves the hands, and the lung 
is the primary site for approximately $50 \%$ of these cases. ${ }^{2}$ Other potential sources are the breast and kidney, and rare sources of tumor include the gastrointestinal system and sarcomas.

The mechanisms for the deposition of tumor cells in the hands are not well defined. Some theories include hematologic dissemination and implicate chemotactic factors, such as prostaglandins, as the means by which malignant cells migrate and adhere to the skeleton. ${ }^{2}$ After adherence, tumor derived factors, including transforming growth factor-B (TGF-B) and insulin-like growth factor 1 (IGF1, alter the balance of osteoclast and osteoblast activities. ${ }^{4}$ Growth factors bind to the receptors on the tumor cell surface and activate autophosphorylation and signaling through pathways that involve cytoplasmic mediators and mitogen- activated protein kinase. Extracellular calcium binds and activates a calcium pump. Signaling through these pathways promotes tumor cell proliferation and additional parathyroid hormone related peptide (PTHrP) production which continues the process of osteoclastogenesis and osteolysis. PTHrP activates osteoblasts to produce receptor activators of nuclear factor NF-KB ligand (RANKL) and downregulate osteoprotegerin (OPG), activating osteoclast precursors and causing osteolysis. ${ }^{3}$ Minor trauma may contribute to the pathogenesis since recent literature indicates the right hand, which is usually the dominant hand, more frequently develops acrometastasis. Repeated unnoticed trauma could lower local tissue resistance which could increase malignant cell implantation.

Physicians often do not suspect acrometastasis. First, this diagnosis is rare. ${ }^{5-9}$ Second, these patients typically present with swelling, erythema, tenderness or loss of function, suggesting a long list of possible diagnoses. Most commonly this presentation suggests an infectious process. Patients then receive antibiotics with either a partial response or no response. The differential diagnoses also include cellulitis, osteomyelitis, and paronychia secondary to bacterial or herpes simplex virus infection. If the patient has a prolonged history of a skin lesion, fungal infection should be considered. Chronic granulomatous infiltration from mycobacterial infection or sar- coidosis can have this presentation. Non-infectious causes include contact dermatitis and benign tumors. Given the bony lytic lesion in our patient, the latter would include epidermoid inclusion cysts, aneurysmal bone cysts, enchondromas, glomus tumors, fibromas, angiomas, and granulomas. Primary tumors, such as squamous cell carcinoma and multiple myeloma, can cause lytic bone lesions. In addition, subungual and giant acral melanomas may present as ulcerating lesions on the thumb with bony lytic involvement. ${ }^{10} \mathrm{Fi}-$ nally, a fungating thumb lesion is very rarely the chief complaint of an undiagnosed lung cancer as was the case with our patient, making acrometastasis hard to suspect and therefore difficult to diagnose.

The best treatment option for acrometastasis is radical surgery. Amputation is the preferred method of treatment in most cases since they present with lesions in the terminal digit. ${ }^{2}$ Systemic chemotherapy or radiation can be used in cases in which amputation causes the patient to lose hand function and in cases with proximal lesions or multiple lesions. ${ }^{2,4,7,8}$ Palliative short course radiation can provide pain relief and help maintain normal hand function. Unfortunately, regardless of the type of treatment, the prognosis of these patients is very poor because acrometastasis generally accompanies widespread disease. Based on a limited number of case reports, the mean survival of these patients is six months or less. Our patient underwent right thumb amputation. He declined to have any type of specific therapy including chemotherapy or radiation and finally consented to hospice care.

\section{KEY POINTS}

1 Suspect acrometastasis in patients who have risk factors for malignancy or who have a poor response to the initial treatment plan.

2 The presence of acrometastasis in patients with cancer helps stage the disease and usually indicates that the prognosis is very poor. 
Author Affiliation: Kamonpun Ussavarungsi is a fellow in pulmonary and critical care medicine at the Mayo Clinic Florida, 4500 San Pablo Road, Jacksonville, FL 32224. Brenda Watkins is a medical student at TTUHSC. Michael Phy is a general internist at TTUHSC.

Received: $01 / 14 / 2012$

Accepted: 01/31/2012

Reviewers: Kenneth Nugent, MD, Rishi Raj, MD

Published electronically: 01/31/2013

Conflict of Interest Disclosures: None

\section{REFERENCES}

1. Stathopoulos GP, Rigatos SK. Rare site of metastasis of non small cell lung cancer. J BUON 2010; 15: 189-90.

2. Flynn CJ, Danjoux C, Wong J, et al. Two cases of acrometastasis to the hands and review of the literature. Curr Oncol 2008; 15: 51-58.

3. Roodman GD. Mechanism of bone metastasis. $N$ Eng $J$ Med 2004; $350: 1655-64$.

4. Berenson JR, Rajdev L, Broder M. Pathophysiology of bone metastases. Cancer Biol Ther2006; 5: 1078-1081.

5. Akjouj S, El Kettani N, Semlali S, et al. Thumb acrometastasis revealing lung adenocarcinoma: a case report with review of literature. Chir Main 2006; 25: 106-8.

6. Nakamura H, Shimizu T, Kodama K, Shimizu H. Metastasis of lung cancer to the finger: a report of two cases. Int $J$ Dermatol2005; 44: 47-9.

7. Carvalho Hde A, Tsai PW, Takagaki TY. Thumb metastasis from small cell lung cancer treated with radiation. Rev Hosp Clin Fac Med Sao Paulo 2002; 57: 283-6.

8. Vijayakumar S, Creditor M. Metastasis to the hand. J Natl Med Assoc 1986; 78: 441-2.

9. Madjidi A, Cole P, Lauricia R. Digital Acrometastasis: a rare initial sign of occult primary squamous cell carcinoma. $J$ Plast Reconstr Aesthet Surg 2009: 62: e365-67.

10. Kim JH, Jeong SE, Shin JB, et al. Giant acral melanoma on the left thumb of a Korean patient. Ann Dermatol 2009; 21(2): $171-4$. 